\title{
Barrett's esophagus is the precursor of all esophageal adenocarcinomas
}

Kit Curtius ${ }^{1}$, Joel H. Rubenstein ${ }^{2,3}$, Amitabh Chak ${ }^{4}$, John M. Inadomi ${ }^{5}$

${ }^{1}$ Centre for Genomics and Computational Biology, Barts Cancer Institute, Queen Mary University of London, London, United Kingdom

${ }^{2}$ Barrett's Esophagus Program, Division of Gastroenterology, University of Michigan, Ann

Arbor, Michigan, USA

${ }^{3}$ Center for Clinical Management Research, Ann Arbor Veterans Affairs Medical Center, Ann Arbor, Michigan, USA

${ }^{4}$ Division of Gastroenterology and Liver Diseases, Case Western Reserve University, Cleveland, Ohio, USA

${ }^{5}$ Division of Gastroenterology, University of Washington, Seattle, Washington, USA

Corresponding author information: k.curtius@qmul.ac.uk

John Vane Science Centre, Charterhouse Square

Barts Cancer Institute

London EC1M 6BQ, United Kingdom

Word count: 2,272 excluding title page, abstract, summary box, references, and figures

\section{Author Contributions:}

KC - Study concept and design; acquisition of data; analysis and interpretation of data; drafting of the manuscript; critical revision of the manuscript for important intellectual content; statistical analysis; obtained funding JHR - acquisition of data; analysis and interpretation of data; drafting of the manuscript; critical revision of the manuscript for important intellectual content AC - Study concept and design; analysis and interpretation of data; drafting of the manuscript; critical revision of the manuscript for important intellectual content JMI - Study concept and design; acquisition of data; analysis and interpretation of data; drafting of the manuscript; critical revision of the manuscript for important intellectual content; obtained funding 


\section{Abstract \\ Objective}

Barrett's esophagus (BE) is a known precursor to esophageal adenocarcinoma (EAC) but current clinical data have not been consolidated to address whether $B E$ is the origin of all incident EAC, which would reinforce evidence for BE screening efforts. We aimed to answer whether all expected prevalent BE, diagnosed and undiagnosed, could account for all incident EACs in the US cancer registry data.

\section{Design}

We used a multi-scale computational model of EAC that includes the evolutionary process from normal esophagus through BE in individuals from the US population. The model was previously calibrated to fit SEER cancer incidence curves. Here we also utilized age- and sexspecific US census data for numbers at-risk. The primary outcome for model validation was the expected number of EAC cases for a given calendar year. Secondary outcomes included the comparisons of resulting model-predicted prevalence of $\mathrm{BE}$ and $\mathrm{BE}$-to-EAC progression to the observed prevalence and progression rates.

\section{Results}

The model estimated the total number of EAC cases in 2010 was 9,970 (95\% Cl 9,140 $11,980)$, which recapitulates all EAC cases from population data. The model simultaneously predicted 8-9\% BE prevalence in high-risk males age 45-55, and 0.1-0.2\% non-dysplastic BEto-EAC annual progression in males, consistent with clinical studies.

\section{Conclusion}

There are no additional EAC cases that plausibly arise in the US population outside the BE pathway. Effective screening of high-risk patients could capture the majority of population destined for EAC progression and decrease mortality through early detection and curative removal of small (pre)cancers during surveillance.

\section{Summary Box}

\section{What is already known about this subject?}

- Barrett's esophagus (BE) patients have a 40 to 50 -fold higher risk of developing esophageal adenocarcinoma (EAC) than the general population yet many remain undiagnosed.

- Identified BE patients receiving surveillance can have early cancers discovered endoscopically, which decreases the high overall EAC-associated mortality.

- Currently around $90 \%$ of patients who develop EAC were never part of a BE surveillance program, and those $B E$ patients on surveillance have a low annual progression rate of $0.1-0.3 \%$ to develop EAC.

\section{What are the new findings?}

- By applying a model that incorporates the evolution from normal cells to BE to EAC in patients, we found that the numbers add up - the expected number of EAC cases in the US population are explained by the published rates of BE described above.

- We cohesively examined the published estimates to determine that all EAC likely arises from both identified BE and occult, undiagnosed BE in the population.

\section{How might it impact on clinical practice in the foreseeable future?}

- Based on current best estimates, our findings suggest there is no public health need to seek cases of a non-BE alternative pathway to EAC.

- Increasing efforts for effective, sensitive screening and surveillance of the true BE population will decrease EAC mortality in the coming years. 


\section{Introduction}

Esophageal adenocarcinoma (EAC) is typically diagnosed when a patient presents with symptoms such as dysphagia. Unfortunately, the majority of these patients do not live past the first year of their diagnosis because by the time dysphagia develops, metastatic cancer is already present. In order to prevent this cancer or detect it at an earlier, more treatable stage, efforts are now made to identify patients with Barrett's esophagus (BE), the only known precursor to EAC. Identified BE patients are believed to have a $\mathbf{4 0}$ to $\mathbf{5 0}$-fold higher annual incidence of EAC than the general population. ${ }^{1}$ Metaplastic BE progresses through dysplasia to cancer. Advances in endoscopic eradication therapy for dysplastic $B E$ discovered during surveillance of BE can now prevent cancer. ${ }^{2}$ However, most cancers arise in patients without previously diagnosed BE suggesting either inadequate screening strategies or, as a recent study proposes, the possible existence of a pathway independent from the BE pathway. ${ }^{3}$ In this study we seek to answer a simple question about the unseen origins of EAC: does overall EAC incidence reflect the number of cancers that would be expected to arise from prevalent $B E$ ? In other words, do any EAC cases remain unaccounted for that ergo did not arise from the typical Barrett's precursor pathway? The answer to this question will importantly guide research and public health efforts. If $B E$ is the major or only precursor of EAC, then investigators should continue to focus on improving $B E$ detection. If $B E$ is not the major precursor of $E A C$ then research needs to focus on identifying alternative pathways and $B E$ screening programs will have limited impact on prevention and early detection of EAC.

In reality, very few individuals who have BE are ever offered an upper endoscopy, and therefore most BE remains asymptomatic and undiagnosed. Patients with gastroesophageal reflux disease (GERD) are technically the only sub-population of the general public typically recommended $\mathrm{BE}$ screening because it is believed they have a $\mathbf{5}$-fold relative risk of developing long segment $B E,{ }^{4}$ yet even so only about $10 \%$ of GERD patients will receive an endoscopy. ${ }^{1}$ This indicates under-screening, likely because patients either do not complain of their GERD symptoms, they respond adequately to medical therapy, or were otherwise not deemed suitably high-risk by their physician to warrant an esophagogastroduodenoscopy (EGD). Nonetheless, the prevalence of BE in the general population is $\mathbf{1 - 2} \%$, whether diagnosed or not ${ }^{5,6}$, and this is likely considerably higher in certain at-risk groups in the US. ${ }^{7-10}$ The main concern is that the average rate to develop EAC in these patients is low - around $\mathbf{0 . 3 \%}$ per year. ${ }^{11}$ Therefore, the majority of endoscopies are futile in finding EAC. We aimed to answer whether all prevalent BE we expect to find, diagnosed and undiagnosed in the US population, could account for all the incident EACs we expect to find as progression rates would imply, to fit the national cancer registry data.

\section{Methods}

The question above is too complex to answer on the 'back of an envelope' because published average rates of progression are dependent on age, birth cohort, and calendar year. In particular for EAC, age-specific incidence rates vary drastically between men and women. ${ }^{12}$ This complexity of timescales involved in normal to premalignant BE to EAC progression has necessitated the creation of quantitative models that analyze cancer 
incidence rates, and project these trends into the future for public health risk assessments and planning. ${ }^{13}$ Models also quantify the potential impact of progression rates measured in clinical studies on hypothetical intervention and surveillance scheduling in efficacy and costeffectiveness studies. ${ }^{14-16}$ Such models allow us to perform quantitative, comparative analyses on the benefits versus harms of proposed screening and surveillance protocols against watch-and-wait strategies; these simply cannot be done heuristically due to the complex nature of cancer evolution.

In this study we model both the onset of $B E$ and the progression of BE to EAC. As a brief background, the multistage clonal expansion for EAC (MSCE-EAC) model is a stochastic model for development of EAC during patient lifetime that includes probabilities of developing $B E$ at various ages, followed by initiation of dysplastic and malignant cell clones in BE with parameters for growth and progression of individual clones to cancer (Figure 1). The inputs only include GERD prevalence (calibrated to age- and sex- specific estimates ${ }^{17,18}$ ) and EAC age- and sex- specific incidence curves provided by SEER registry. ${ }^{12}$ The BE prevalence and neoplastic progression rates are calibrated to fit those inputs, i.e. they are not based on observed BE prevalence or neoplastic progression rates from empiric studies. Briefly, the model includes GERD-stratified risk curve to develop $B E$, which is modeled as an age-dependent rate of exponential $B E$ onset each calendar year with an unknown baseline parameter $v_{0}$. The patient-specific BE lengths can vary, derived from a Beta distribution with general population mean length set to $2-3 \mathrm{~cm}$. Beyond $v_{0}$, the baseline constant rate for $B E$ onset, the additional model parameters govern the evolutionary dynamics for dysplastic and malignant growth and EAC detection. The model parameters have been previously calibrated such that the resulting hazard functions fit to EAC age- and sex-specific incidence curves provided by SEER registry. ${ }^{13}$ We found during rigorous model selection with likelihood ratio tests that models stratified by birth cohort and sex best fit the incidence data, robust to sensitivity analyses (Figure 2). With these fits, the model outputs used for this study include the expected number of EAC cases in an at-risk population at a given year calculated using the hazard function $h_{E A C}$ (see Supplementary material for equation details), along with the $B E$ prevalence and the resulting BE-to-EAC progression rates (predicted as specific to age, sex, and birth cohort).

This model has been used and improved in comparative analyses within the $\mathrm{NCl}$ Cancer Intervention and Surveillance Modeling Network (CISNET) consortium for the past 9 years, which has enabled numerous studies on sensitivity of biopsy sampling techniques for detection of small dysplastic lesions ${ }^{14}$, on influence of patient-specific molecular BE dwell time on future EAC risk ${ }^{19}$, and on cost-effectiveness of endoscopic eradication therapy for certain BE risk groups during surveillance. ${ }^{15}$ In our original study on modeling EAC incidence and mortality rates from 1975-2010, we used SEER-specific model fits combined with US census data to estimate past and predict future EAC-related deaths but did not include predicted EAC cases by calendar year when applied to US census data. ${ }^{13}$

In the Results below, we expand upon prior modeling to help elucidate an answer to our general public health question - "Is BE the precursor of all EAC?" To do this, we first applied the model to estimate the number of EAC cases using the US age- and sex- specific at-risk population estimates from the US census data, to be able to compare with the expected number quoted by Vaughan and Fitzgerald. ${ }^{1}$ This outcome serves as an independent 
validation of successful calibration of our model to EAC incidence. Then we compared the simultaneous predictions of age-specific BE prevalence using the MSCE-EAC model with the published data currently used for screening rationale, ${ }^{20}$ which included endoscopic reports from the Clinical Outcomes Research Initiative (CORI) for more than 150,000 patients, most of whom were born around 1950. We also compared the mathematical predictions of neoplastic progression rate from non-dysplastic Barrett's esophagus to published estimates.

\section{Results}

First, Vaughan and Fitzgerald estimated that the newly diagnosed number of cases for ages greater than 40 to be roughly around 10,000 total in the US every year based on data from 2010 with an average EAC incidence rate across all age groups. ${ }^{1}$ With the Markov model framework we can analytically compute the EAC hazard function and estimate the expected number of newly diagnosed EAC cases by age and year separately for men and women when considering also population data. As a starting point using 2010 census person-year data, ${ }^{21}$ the model predicts about 2.2 million adults had prevalent BE in 2010, which is around $1.6 \%$ of the general US population over age 40 . Then, for age groups greater than 40 in both sexes of all races, our single-age calibrated model estimated that the expected number of new EAC cases diagnosed in 2010 was equal to 9,970 (95\% Cl 9,140-11,980).

We also computed the analogous estimate for EAC cases using incidence rates quoted directly from the SEER registry for ages 40-90, which was found to be 9,400 EAC cases total in 2010. ${ }^{12}$ Thus, the estimate generated by our computational model of progression from $\mathrm{BE}$ to EAC is consistent with the total number of EAC cases reported in SEER, which also aligns with the $10 \mathrm{~K}$ incident cases quoted by Vaughn and Fitzgerald. ${ }^{1}$

Secondly, we considered what the model simultaneously predicted for BE prevalence and BE-to-EAC progression rates in order to achieve the expected $10 \mathrm{~K}$ cases. Breaking down the contributions of the 2.2 million total BE patients estimated above, the model predicted BE prevalence to be $1.9 \%-2.4 \%$ in men and $0.4 \%-0.5 \%$ in women in the general US population ages 45-55 in 2010 (Figure 3A). These predictions concur with best estimates ${ }^{5,6}$ and influence the total EAC cases predicted by the multi-stage model. To further explore implications for high-risk patients, we note the model predicted a BE prevalence of $7.9 \%$ 9.3\% in US men with symptomatic GERD who are cancer-free ages 45-55 in 2010 when the relative risk (RR) of $B E$ versus non-GERD individuals is assumed to be $R R=5$ (Figure 3B). This is also consistent with the estimate of $8 \%$ provided by Vaughan and Fitzgerald ${ }^{1}$ for prevalence of cancer-free BE diagnoses amongst GERD patients who undergo an upper endoscopy. Further, the model's predicted age-specific BE prevalence curves by sex were consistent with previous results on BE prevalence from the Clinical Outcomes Research Initiative (CORI) study ${ }^{20}$ (Figure $3 \mathrm{~A}$ ). Compared to our model results and $8 \%$ quoted above ${ }^{1}$ for high-risk groups, the CORI study independently found similar BE prevalence in white men with GERD of $6.3 \%$ for ages $40-49$ and $9.3 \%$ for ages $50-59$ (Figure 3B). To account for likely heterogenous relative risk of developing BE in GERD populations based on symptom onset age, BE length, and other factors, ${ }^{4,22-26}$ we also considered a range of fixed values $(\mathrm{RR}=2-6)$ and found age-specific trends broadly consistent to overall BE prevalence results in CORI. Observed BE prevalence in white women undergoing screening was less precise in the CORI study data yet still coincided with our predictions for women (Figure 3B). 
Lastly, we previously found using this model that, for individuals born after 1940, the range of progression rates from BE-to-EAC was $0.10 \%$ to $0.20 \%$ for men, and this was about twice as high as we found for women. ${ }^{13}$ These are plausibly low rates compared to current best estimates. ${ }^{11,27}$ If there were a significant alternate non-BE pathway for EAC development, then this model (which does not include a non-BE pathway) would have estimated either a lower predicted population incidence of EAC than what was observed in SEER, a greater prevalence of $B E$ than what was observed, or a greater rate of neoplastic progression among non-dysplastic BE than observed.

\section{Discussion}

Based on the published epidemiology of BE and EAC, our analysis suggests that a major alternative non-BE pathway to EAC is an unlikely scenario. The existence of such an alternative pathway was suggested by a retrospective analysis of macroscopic reports of EAC specimens diagnosed without BE in two cohorts from the US and UK by Sawas and colleagues; however, their study conclusions remain speculative due to some important limitations including 1) a lack of longitudinally followed cases to EAC from non-BE patient esophageal tissue, and 2) the plausibility that small BE segments were completely overtaken by malignant expansions and thus were unmeasurable at cancer diagnosis. ${ }^{3}$ Moreover our result that $B E$ is the main origin of $E A C$ does not necessarily refute the existence of differing phenotypes for EAC - the finding that presence of BE was associated with better survival could plausibly be explained by the theory that more aggressive cancers are likely to replace the precursor BE more readily than less aggressive cancers.

Indeed, genetic and epigenetic analyses have also consistently shown BE and EAC to be very similar, ${ }^{28-31}$ and one study that sought genomic differences between adenocarcinomas with and without BE failed to reveal molecular differences between the two. ${ }^{32}$ Nonetheless, this is fortunate news that, with adequate uptake, screening for BE by upper endoscopy or

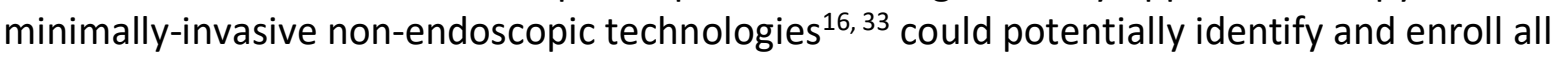
patients who are at risk for developing EAC into a surveillance program. Practically speaking, this would require very sensitive screening to detect all prevalent BE of any size and location (such as small BE patches at irregular Z-lines) but our results suggest these patients would constitute the majority of future EAC cases if detected.

Although the overall progression to $E A C$ is low in patients diagnosed with $B E$, for those selected $B E$ patients who have high grade dysplasia and/or early EAC detected during surveillance, effective treatment can save lives. In this way, our analysis reinforces the primary goal in BE screening for EAC prevention - that potentially all mortality caused by $E A C$ in the general population could be prevented by effective surveillance of the entire $B E$ population. Further, by mathematically analyzing the time-dependent nature of cumulative risk of BE in GERD patients, we can also use our multi-stage model framework to improve identification of at-risk populations by optimizing the timing of initial screening recommended for BE in symptomatic GERD. ${ }^{34}$ Although current intensive 'one-size-fits-all' surveillance strategies ${ }^{35-40}$ would lead to high costs for those over-diagnosed BE screen cases and surveillance strategies clearly need to improve, we conclude that there is a strong rationale for screening for BE to reduce EAC mortality. 


\section{Figures}

Figure 1: The stochastic, multi-scale model for EAC development (MSCE-EAC) includes conversion from normal squamous epithelium in the esophagus to $B E$ metaplasia with $B E$ onset rate $v(t)$, which is a function of a baseline rate $v_{0}$ and age-dependent prevalence of GERD $p_{G E R D}(t)$ (see Methods for details). Two-hit processes with rates $\mu_{0}, \mu_{1}$ can initiate a premalignancy (e.g., inactivation of tumor suppressor gene TP53 in non-dysplastic BE due to mutation/copy number alteration in a BE daughter cell creates first cell of a high grade dysplasia lesion). Premalignant cell growth rates are defined as $\alpha_{p}=$ division rate, $\beta_{p}=$ death/ differentiation rate per year. Malignant transformation with rate $\mu_{2}$ creates the first cell of a pre-clinical clone that can grow with rates $\alpha_{M}=$ division rate, $\beta_{M}=$ death/differentiation rate per year. Size-based probability $\rho$ for detection of preclinical malignant clone can lead to patient-specific time of incident EAC.

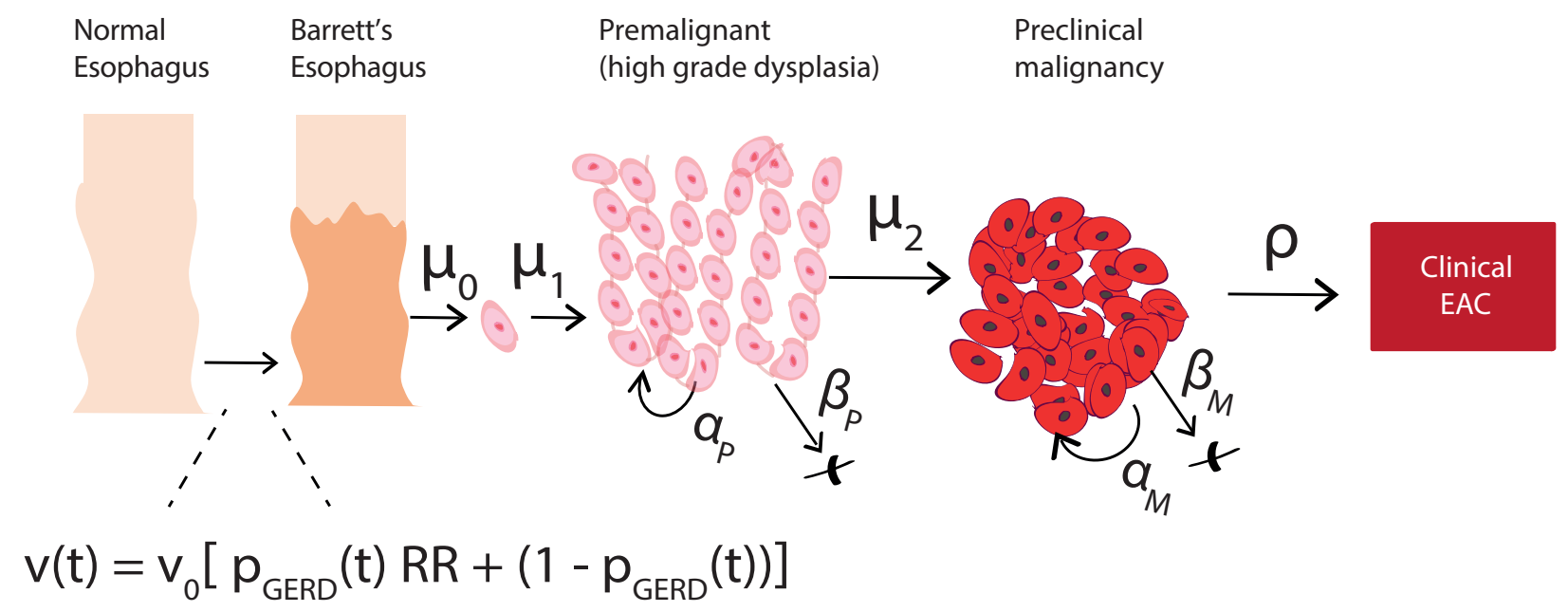

Figure 2: MSCE-EAC model was previously calibrated to EAC incidence curves (solid lines), stratified by sex and birth cohort, for consistency with SEER data trends (dashed lines).

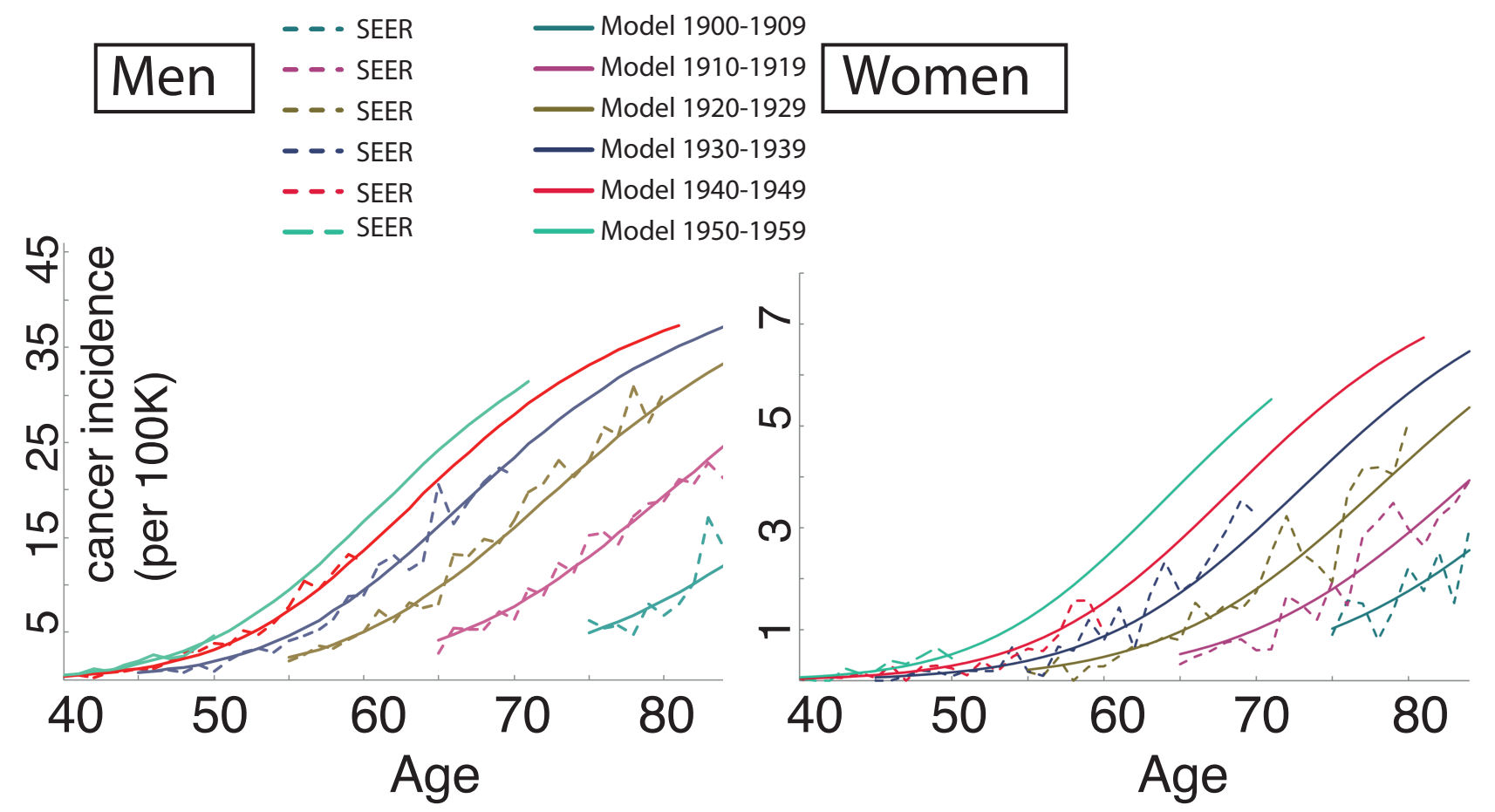


Figure 3: Model predictions for BE-positive yield in a cancer-free population (solid lines) are consistent with observed data (dashed lines) from Clinical Outcomes Research Initiative (CORI). ${ }^{20}$ (A) Solid lines show model results for the general US population stratified by sex from the 1950 birth cohort, with contributions of relative risk (RR) of BE from the agespecific, prevalent GERD population assumed to be $R R=5$ (shaded areas, $R R=[2,6]$ ). Dashed lines show consistency with observed BE prevalence data for patients without indication for screening in CORI, which are independent of the model. Model BE prevalence estimates are part of the evolutionary multi-stage process and thus affect predictions of the total EAC cases predicted (see Results). (B) Solid lines show model results for the symptomatic GERD subpopulation stratified by sex from the 1950 birth cohort with relative risk for BE set to $R R=5$. The shaded areas are predicted ranges for GERD subpopulations with fixed $R R=2-6$ to describe a wide range of increased risks of $B E$ in published estimates, based on factors such as onset age of GERD and BE length. The true GERD-specific BE prevalence contributing to mathematical formulation used in $(A)$ is within this region, where individual contributions are based on GERD onset age and underlying distribution of RR. Dashed lines show BE prevalence data for patients with GERD, and/or another indication for screening, in CORI.

A

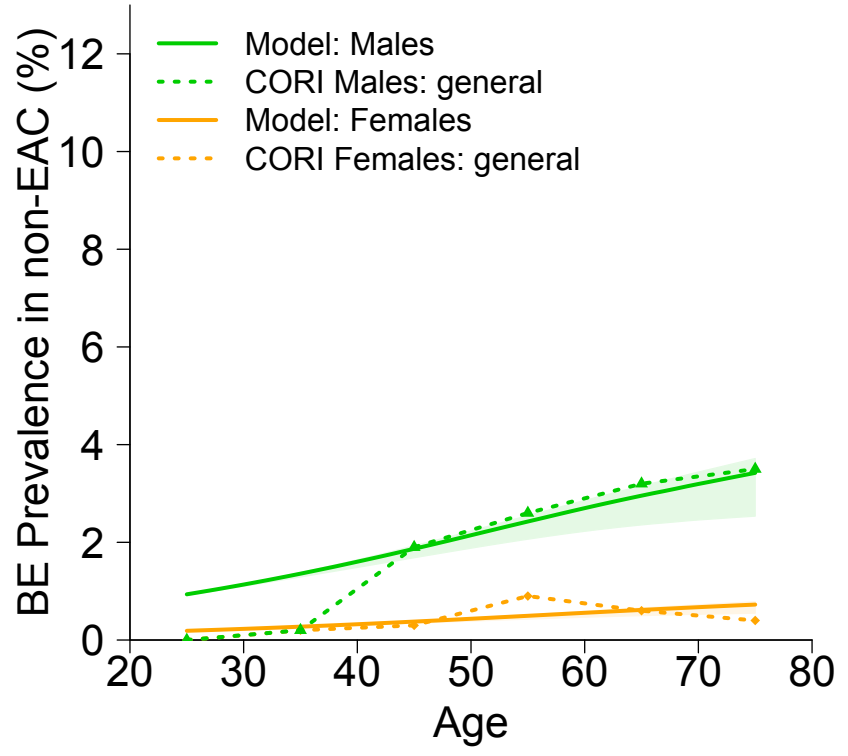

B

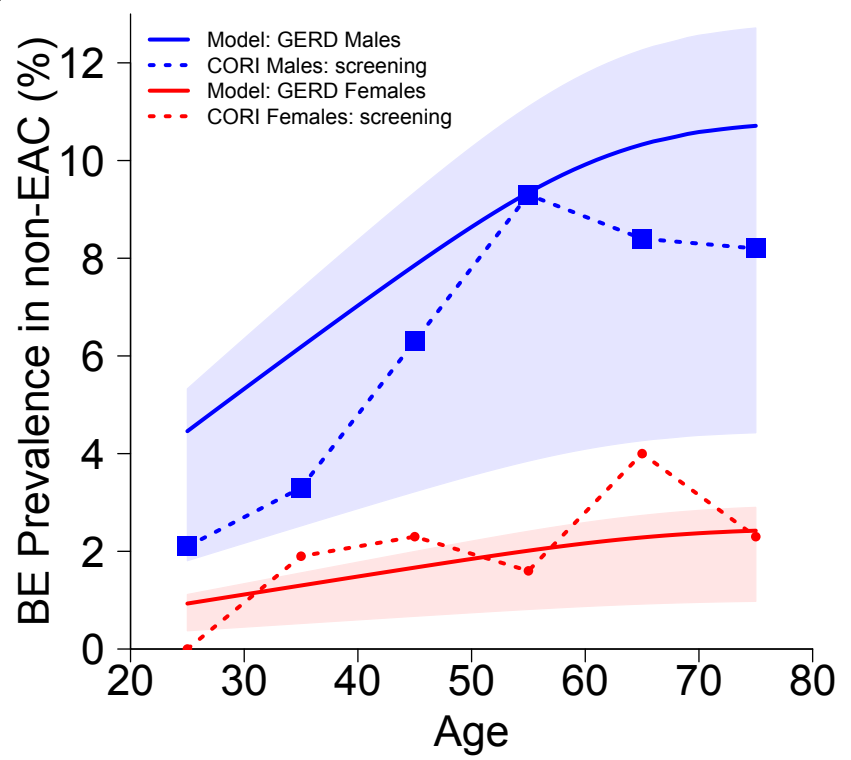

Data sharing statement

All data used in our analysis are publicly available. ${ }^{12,20}$ All equations are provided either in

Figures and Supplementary material or were previously published along with model parameters. ${ }^{13,14}$ Code to solve equations was developed in $\mathrm{R}$ (version 3.6.1). Scripts to repeat our analysis will be made available at: github.com/yosoykit/BEtoEAC_Results.

Conflict of interest statement:

$\mathrm{KC}, \mathrm{JHR}$, and JMI declare no potential conflicts of interest.

$A C$ has founders shares and stock options in LucidDx, serves as a consultant to LucidDx, has sponsored research with LucidDx, and has a royalty interest in patents licensed to LucidDx. $\mathrm{He}$ is also a consultant for Interpace Diagnostics and receives research support from C2 Therapeutics/Pentax Inc. 


\section{Acknowledgments:}

The authors thank the Clinical Outcomes Research Initiative (CORI) study for access to endoscopy data. This work was supported by funding from NIH grants CISNET U01 CA152926, U01 CA199336 (JMI, KC, JHR), K24 DK080941 (JMI, KC), and a UKRI Rutherford Fund Fellowship (KC). JHR is also supported by NIH grant U54 CA163059 (BETRNET). AC is supported by NIH grants U54 CA163060 and P50 CA150964.

\section{References}

1. Vaughn TL, Fitzgerald RC. Precision prevention of oesophageal adenocarcinoma. Nat Rev Gastroenterol Hepatol 2015;12:243-248.

2. Standards of Practice C, Wani S, Qumseya B, et al. Endoscopic eradication therapy for patients with Barrett's esophagus-associated dysplasia and intramucosal cancer. Gastrointest Endosc 2018;87:907-931 e9

3. Sawas T, Killcoyne S, Iyer PG, et al. Identification of Prognostic Phenotypes of Esophageal Adenoacarcinoma in 2 Independent Cohorts. Gastroenterology 2018;155(6):1720-1728.

4. Taylor JB, Rubenstein JH. Meta-analyses of the effect of symptoms of gastroesophageal reflux on risk of Barrett's esophagus. Am J Gastroenterology 2010;105:1730-1737.

5. Zagari RM , Fuccio L , Wallander MA, et al. Gastro-oesophageal reflux symptoms, oesophagitis and Barrett's oesophagus in the general population: the LoianoMonghidoro study. Gut $2008 ; 57: 1354-9$.

6. Ronkainen J, Aro P, Storskrubb T, et al. Prevalence of Barrett's esophagus in the general population: an endoscopic study. Gastroenterology 2005; 129 : 1825 - 31 .

7. Ward EM, Wolfsen HC, Achem SR, et al. Barrett's esophagus is common in older men and women undergoing screening colonoscopy regardless of reflux symptoms. Am J Gastroenterol 2006;101: $12-7$.

8. Rex DK, Cummings OW, Shaw $M$, et al. Screening for Barrett's esophagus in colonoscopy patients with and without heartburn. Gastroenterology 2003; 125 : $1670-7$.

9. Gerson LB, Shetler K, Triadafilopoulos G. Prevalence of Barrett's esophagus in asymptomatic individuals. Gastroenterology 2002; $123: 461-7$.

10. Rubenstein $\mathrm{JH}$, Morgenstern $\mathrm{H}$, Appelman $\mathrm{H}$, et al. Prediction of Barrett's esophagus among men. Am J Gastroenterol 2013; 108:353-362.

11. Kroep S, Lansdorp-Vogelaar I, Rubenstein JH, et al. An accurate cancer incidence in Barrett's esophagus: a best estimate using published data and modeling. Gastroenterology. 2015;149(3): 577-585.

12. SEER*Explorer: An interactive website for SEER cancer statistics [Internet]. Surveillance Research Program, National Cancer Institute. [Cited 2019 Apr 15]. Available from https://seer.cancer.gov/explorer/.

13. Kong CY, Kroep S, Curtius K, et al. Exploring the recent trend in esophageal adenocarcinoma incidence and mortality using comparative simulation modeling. Cancer Epidemiol Biomarkers Prev 2014;23(6):997-1006.

14. Curtius K, Hazelton WD, Jeon J, et al. A Multiscale Model Evaluates Screening for Neoplasia in Barrett's Esophagus. PLoS Comput Biol 2015;11(5):e1004272. 
15. Kroep S, Heberle CR, Curtius K, et al. Radiofrequency Ablation of Barrett's Esophagus Reduces Esophageal Adenocarcinoma Incidence and Mortality in a Comparative Modeling Analysis. Clin Gastroenterol Hepatol 2017;15(9):1471-1474.

16. Heberle CR, Omidvari AH, Ali A, et al. Cost Effectiveness of Screening Patients with Gastroesophageal Reflux Disease for Barrett's Esophagus with a Minimally Invasive Cell Sampling Device. Clin Gastroenterol Hepatol. 2017 Sep;15(9):1397-1404.e7

17. Ruigómez A, García Rodríguez LA, Wallander MA, et al. Natural history of gastrooesophageal reflux disease diagnosed in general practice. Aliment Pharmacol Ther 2004 Oct 1;20(7):751-60.

18. Ruigómez A, Wallander MA, Lundborg P, et al. Gastroesophageal reflux disease in children and adolescents in primary care. Scand J Gastroenterol 2010;45(2):139-46.

19. Curtius K, Wong CJ, Hazelton WD, et al. A Molecular Clock Infers Heterogeneous Tissue Age Among Patients with Barrett's Esophagus. PLoS Comput Biol 2016 May 11;12(5):e1004919.

20. Rubenstein JH, Mattek N, Eisen G. Age- and sex-specific yield of Barrett's esophagus by endoscopy indication. Gastrointest Endosc 2010;71(1):21-7.

21. United States Census Bureau. Census of Population and Housing [online], http:// www.census.gov/prod/www/decennial.html (2015). Accessed Feb 2020.

22. Thrift AP, Kramer JR, Qureshi Z, et al. Age at onset of GERD symptoms predicts risk of Barrett's esophagus. Am J Gastroenterol 2013;108:915-22.

23. Eisen GM, Sandler RS, Murray S, et al. The relationship between gastroesophageal reflux disease and its complications with Barrett's esophagus. Am J Gastroenterol 1997;92:27-31.

24. Anderson LA, Watson RG, Murphy SJ, et al. Risk factors for Barrett's oesophagus and oesophageal adenocarcinoma: results from the FINBAR study. World J Gastroenterol 2007;13:1585-94.

25. Edelstein ZR, Bronner MP, Rosen SN, et al. Risk factors for Barrett's esophagus among patients with gastroesophageal reflux disease: a community clinic-based case-control study. Am J Gastroenterol 2009;104:834-42.

26. Shaheen N, Ransohoff DF. Gastroesophageal reflux, Barrett esophagus, and esophageal cancer - Scientific review. JAMA 2002;287:1972-81.

27. de Jonge PJF, van Blankenstein $\mathrm{M}$, Looman CWN, et al. Risk of malignant progression in patients with Barrett's oesophagus: a Dutch nationwide cohort study. Gut 2010;59:103-1036.

28. Ross-Innes CS, Becq J, Warren A, et al. Whole-genome sequencing provides new insights into the clonal architecture of Barrett's esophagus and esophageal adenocarcinoma. Nat Genet 2015 Sep;47(9):1038-1046.

29. Secrier M, Li X, de Silva N, et al. Mutational signatures in esophageal adenocarcinoma define etiologically distinct subgroups with therapeutic relevance. Nat Genet 2016 Oct;48(10):1131-41.

30. Yu M, Maden SK, Stachler M, et al. Subtypes of Barrett's oesophagus and oesophageal adenocarcinoma based on genome-wide methylation analysis. Gut 2018 Jun 8. doi: 10.1136/gutjnl-2017-314544.

31. Liu Y, Sethi NS, Hinoue T, et al. Comparative Molecular Analysis of Gastrointestinal Adenocarcinomas. Cancer Cell 2018 Apr 9;33(4):721-735. 
32. Ferrer-Torres D, Nancarrow DJ, Kuick R, et al. Genomic similarity between gastroesophageal junction and esophageal Barrett's adenocarcinomas. Oncotarget 2016 Aug 23;7(34):54867-54882. doi: 10.18632/oncotarget.10253.

33. Moinova HR, LaFramboise T, Lutterbaugh JD, et al. Identifying DNA methylation biomarkers for non-endoscopic detection of Barrett's esophagus. Sci Transl Med 2018 Jan 17;10(424)

34. Curtius K, Dewanji A, Hazelton WH, et al. Optimal timing for cancer screening and adaptive surveillance using mathematical modeling. bioRxiv 2020 doi: https://doi.org/10.1101/2020.02.11.927475

35. Codipilly DC, Chandar AK, Singh S, et al. The Effect of Endoscopic Surveillance in Patients With Barrett's Esophagus: A Systematic Review and Meta-analysis. Gastroenterology. 2018;154(8): 2068-2086.

36. American Gastroenterological Association, Spechler SJ, Sharma P, et al. American Gastroenterological Association medical position statement on the managementof Barrett's esophagus. Gastroenterology 2011; 140:1084-1091.

37. Shaheen NJ, Falk GW, lyer PG, et al; American College of Gastroenterology. ACG Clinical Guideline: Diagnosis and Management of Barrett's Esophagus. Am J Gastroenterol 2016 Jan;111(1):30-50

38. Fitzgerald RC, di Pietro M, Ragunath K, et al; British Society of Gastroenterology. British Society of Gastroenterology guidelines on the diagnosis and management of Barrett's oesophagus. Gut 2014 Jan;63(1):7-42.

39. di Pietro M, Fitzgerald RC; BSG Barrett's guidelines working group. Revised British Society of Gastroenterology recommendation on the diagnosis and management of Barrett's oesophagus with low-grade dysplasia. Gut 2018 Feb;67(2):392-393.

40. Qumseya B, Sultan S, Bain P, et al; ASGE Standards of Practice Committee Chair. ASGE guideline on screening and surveillance of Barrett's esophagus. Gastrointest Endosc 2019 Sep;90(3):335-359.e2. 